\title{
A new ultrasound sign in the diagnosis of pediatric maxillary sinusitis
}

\author{
Ahmad Ghasemi ${ }^{1}$, Atabak Allafasghari ${ }^{1}$, Mani Mofidi ${ }^{2 *}$ \\ Received: 5 Sep 2016 \\ Published: 28 Feb 2018
}

\begin{abstract}
Background: Computed tomography scans (CT scan) and X-rays are used to diagnose paediatric maxillary sinusitis. This study aimed at exploring the diagnostic value of the conventional and colour Doppler ultrasounds and their specific findings in cases of paediatric sinusitis.

Methods: A total of 60 children diagnosed with sinusitis were included in this study. The conventional and colour Doppler ultrasounds of the sinus were performed on each of them. The symptoms that suggested increased blood flow to the sinuses were interpreted as positive findings on the colour Doppler ultrasound and were named "Ghasemi signs" for the purpose of this study. Such symptoms included unilateral artery bumps on the front artery, reduction of arterial resistive index (RI) to less than 0.5 , and diameter of $2 \mathrm{~mm}$ or above for maxillary arteries. Sensitivity, specificity, and positive and negative predictive values, and accuracy of the conventional and colour Doppler ultrasounds were also calculated.

Results: Compared to CT scan, the conventional ultrasound showed sensitivity and specificity of $73.4 \%$ and $100 \%$, respectively. Sensitivity and specificity for the colour Doppler ultrasound were $89.36 \%$ and $100 \%$, respectively. The maxillary artery diameter in normal and affected maxillary sinuses were, respectively, $2.4 \mathrm{~mm}(2.1-2.6,95 \% \mathrm{CI})$ and $1.7 \mathrm{~mm}(1.6-1.9,95 \% \mathrm{CI})$, with $\mathrm{p}<0.001$. The RI of the affected sinuses were $0.47(0.45-0.49,95 \% \mathrm{CI})$, and those of the normal sinuses were $0.58(0.54-0.61,95 \% \mathrm{CI})$, with $\mathrm{p}<0.001$.

Conclusion: The findings of this study revealed that the conventional ultrasound agrees with the CT scans in the diagnosis of paediatric maxillary sinusitis. This diagnostic modality becomes even more valuable when the colour Doppler is used, particularly when considering the specific symptoms (Ghasemi signs) suggested by this study.
\end{abstract}

Keywords: Sinusitis, Pediatric, Ultrasound, Colour Doppler, Ghasemi sign

Copyright $\odot$ Iran University of Medical Sciences

Cite this article as: Ghasemi A, Allafasghari A, Mofidi M. A new ultrasound sign in the diagnosis of pediatric maxillary sinusitis. Med J Islam Repub Iran. 2018(28 Feb);32:16. https://doi.org/10.14196/mjiri.32.16

\section{Introduction}

Rhinosinusitis (inflammation of the mucosa of the nasal cavity and the sinuses at the same time) is a common disease in children $(1,2)$. It usually results from a complication of viral infection of the upper respiratory tract or from an allergic inflammation $(3,4)$. The signs and symptoms of this disease in children are nonspecific and include cough, fever, headache, rhinorrhoea, post-nasal drip, and nasal congestion $(5,6)$. Paediatric rhinosinusitis is diagnosed by 3 methods: the clinical method, nasal endoscopy, and imaging (7-9).

Radiography has been used in the diagnosis of sinusitis. Sinus opacification, air-fluid level, and increased mucus

Corresponding author:Dr Mani Mofidi, manimofidi@yahoo.com

1. Department of Radiology, Imam Khomeini Hospital, Mazandaran University of Medical Sciences, Sari, Iran.

2. Emergency Medicine Department, Rasoul Akram Hospital, Emergency Management Research Center, Iran University of Medical Sciences, Tehran. Iran. thickness are considered to be positive radiological evidences of sinusitis. Various studies have shown moderate sensitivity and specificity of X-rays in diagnosing sinusitis in children and have also noted that the inability of radiography to differentiate among inflammation, infection, and tumour is an important problem in this diagnostic modality (10-12).

CT scan has been introduced as the gold standard in checking sinuses; however, considering the high levels of radiation received by the patients, this diagnostic method should only be used in special circumstances (13).

Ultrasonography is fast, safe, inexpensive, and a readily-

$\uparrow$ What is "already known" in this topic:

Ultrasonography is fast, safe, inexpensive, and a readily-available modality for the evaluation of maxillary sinus in children.

$\rightarrow$ What this article adds:

Ultrasonography is more valuable when the colour Doppler is used, particularly when considering the specific symptoms (Ghasemi signs) suggested by this study. 
available diagnostic method for the evaluation of maxillary sinus (14). A few studies have been conducted in the field of ultrasound and its value in the diagnosis of paediatric sinusitis. Nonetheless, the results achieved from such studies are heterogeneous.

On the other hand, using colour Doppler ultrasound in the case of sinus infection has been suggested for sinusitis maxillary diagnosis. Based on previous studies, it can be concluded that the sound stream can be produced in the nonsuppurative sinus secretions, but not in purulent mucous secretions due to their high viscosity. Unlike nonpurulent sinusitis in purulent sinusitis, the colour Doppler ultrasound also shows increased blood flow. Accordingly, the purulent secretions are distinguishable from the sinus serous secretions $(15,16)$.

Sinusitis is an inflammatory process and inflammation has been associated with increased blood flow (17). Thus, it can be hypothesized that at the time of sinusitis, the arteries carrying blood into the sinuses increase blood flow velocity and diameter to increase the blood flow to the sinuses.

This study was conducted to evaluate the diagnostic value of ultrasounds for paediatric maxillary sinusitis and to introduce new colour Doppler ultrasound findings (Ghasemi signs) for these patients.

\section{Methods}

This study was conducted at a teaching hospital in Sari, Iran, during 2014 and 2015. The study was approved by the ethics committee of the University. After a full explanation of the project and obtaining informed consent from the parents, 60 children were enrolled in the study. Inclusion criteria were as follow: age 4 to 17 years, and having symptoms suggesting rhinosinusitis (cough, fever, headache, rhinorrhoea, post-nasal drip, and nasal congestion).

At first, the sinus CT scan was taken as the gold standard and was evaluated by one radiologist, who was blind to the study. If the evidence of sinusitis, including fluid collection or mucosal thickening, was seen in at least one of the maxillary sinuses, the child was then diagnosed with sinusitis and included in the study. On the same day, sinus ultrasounds were done on the children by another radiologist, who did not know the result of the CT scans. Two radiologists performed the sinus ultrasounds.

A Samsung Medison SonoAce X8 machine, with 5$7 \mathrm{MHz}$ frequency curve linear sector probe, was used for the ultrasound. The ultrasounds were done with children in sitting position, while their hands were on both sides and their head was semi- extended. The ultrasonographic images were taken from the lateral nasal section on the sides below the orbital section by sweeping to the lateral and lower parts of the face to find the sinuses.

\section{Color Doppler ultrasound}

The superior anterior alveolar artery, ie, the maxillary artery branch, supplies the maxillary sinus blood (18). Since measuring the diameter of the subdirectories in living human beings is difficult due to the narrow and sometimes indepth tissue, this study investigated the changes in the di- ameter of the maxillary artery just before it split into subbranches in the anterolateral wall of the maxillary sinus. To the best of our knowledge, to date, no study has been conducted on the diameter of the artery in children. In this study, the diameter of the artery in 20 healthy children was measured, and diameter of less than $2 \mathrm{~mm}$ was considered as normal.

Another investigated parameter was resistive index (RI) of arteries, which was calculated by the colour Doppler ultrasound in normal and involved sinuses.

$\mathrm{RI}=$ (peak systolic flow velocity - end-diastolic flow velocity /peak systolic flow velocity).

The symptoms that indicated an increased blood flow and were interpreted as positive findings in the colour Doppler ultrasonography were referred to as Ghasemi signs in this study. Such symptoms included unilateral artery bumps on the front artery, reduction of RI to less than 0.5 , and an increase of more than $2 \mathrm{~mm}$ in the diameter of the front artery in patients with bilateral involvement. The presence of at least one of these findings was interpreted as increased blood flow in the colour Doppler ultrasonography.

\section{Statistical analysis}

Data were collected and entered into the IBM SPSS 20 software. Chi square test was used to analyse the qualitative data, while t test was used to analyse the quantitative variables.

The total results of the CT scans (as the gold standard method) were compared with those of the ultrasound. Sensitivity, specificity, positive and negative predictive value, and accuracy were calculated according to the available formulae.

On the other hand, the kappa coefficient test was used to determine compliance and agreement between the ultrasound and CT scan in this investigation. Fleiss had proposed the following categories for the strength of agreement for the kappa coefficient: kappas over 0.75 as excellent, 0.40 to 0.75 as fair to good, and below 0.40 as poor (19).

Also, considering the possibility of bone thickness, the results were analysed for 3 separate age groups of 4 to 8 years, 9 to 12 years, and 13 to 17 years.

\section{Results}

The CT scan of sinuses was performed for 73 children with symptoms of sinusitis. Out of the 73 children, 60 with the evidence of fluid collection or mucosal thickening in at least one of the right or left maxillary sinuses were included. The patient flow diagram is presented in Fig. 1. Of the 60 children, 34 were male and 26 female. The mean \pm SD age was $8.25 \pm 4.08$ years. The ultrasound of the maxillary sinuses was done on the same day (total 120 maxillary sinuses). No adverse effects were seen in children after performing ultrasound or CT scan. Based on the results of the CT scans, among the 120 maxillary sinuses of the 60 children examined in this study, 94 maxillary sinuses had sinusitis and 26 were normal (fluid collection: 23 in the right and 26 in the left side and mucosal thickening: 37 in the right and 33 in the left side). The ultrasound correctly diag- 

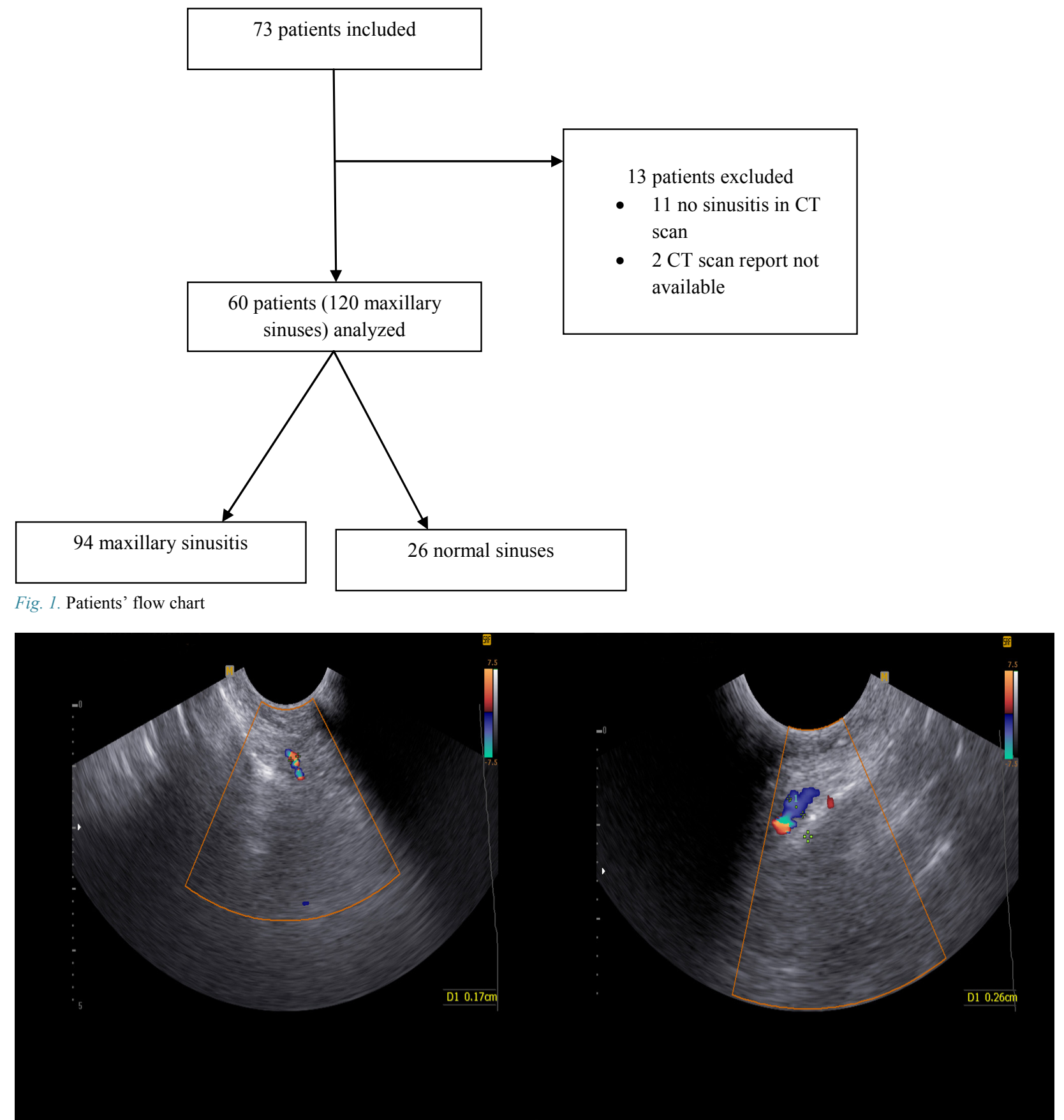

Fig. 2. Normal (left side) and abnormal (right side) diameter of maxillary artery. Diameter more than $2 \mathrm{~mm}$ means an increase in the blood flow to the sinus because of sinusitis (Ghasemi sign).

nosed 69 sinus abnormalities. The kappa coefficient test revealed a moderate agreement between the ultrasound and CT scan in the diagnosis of maxillary sinusitis $(\mathrm{K}=0.545$ and $\mathrm{p}<0.001$ ).

On the other hand, the colour Doppler ultrasound showed 84 instances of increased blood flow or increased diameter of the artery on the affected side, compared to the normal side of maxillary sinuses, and yet had excellent agreement with the CT scan $(\mathrm{K}=0.784$ and $\mathrm{p}<0.001)$. The normal and abnormal diameters of the artery found in the colour Doppler ultrasounds are demonstrated in Fig. 2.

Table 1 shows sensitivity, specificity, positive predictive value, negative predictive value, and accuracy of the conventional ultrasound and the Doppler ultrasound for the diagnosis of sinusitis.

The diameters of maxillary artery in the normal maxillary sinuses and in sinusitis-affected ones were found to be 1.7 $\mathrm{mm}(1.6-1.9,95 \% \mathrm{CI})$ and $2.4 \mathrm{~mm}(2.1-2.6,95 \% \mathrm{CI})$, respectively $(\mathrm{p}<0.001)$.

The RI in normal and involved maxillary sinuses were measured and compared. The RI in the affected sinuses was $0.47(0.45-0.49,95 \% \mathrm{CI})$, while RI was $0.58(0.54-0.61$, $95 \% \mathrm{CI})$ in the normal sinuses, $(\mathrm{p}<0.001)$.

To investigate the possible effects of bone thickness and 


\begin{tabular}{|c|c|c|c|c|c|}
\hline & Sensitivity & Specificity & PPV & NPV & Accuracy \\
\hline Ultrasound & 73.40 & 100 & 100 & 50.98 & 79.16 \\
\hline Doppler & 89.36 & 100 & 100 & 70.58 & 90 \\
\hline
\end{tabular}

PPV: positive predictive value; NPV: negative predictive value

Table 2. Accuracy of conventional ultrasound and Doppler in diagnosis of sinusitis in different ages

\begin{tabular}{|c|c|c|c|c|c|c|c|c|}
\hline \multicolumn{2}{|c|}{ Age (years) } & Sensitivity & Specificity & PPV & NPV & Accuracy & p-value & Kappa \\
\hline $4-8$ & Conventional & 69.70 & 100 & 100 & 28.57 & 72.97 & 0.007 & 0.332 \\
\hline$(n=37)$ & Doppler & 87.87 & 100 & 100 & 50 & 89.18 & $<0.001$ & 0.61 \\
\hline $9-12$ & Conventional & 85.71 & 100 & 100 & 85.71 & 92.30 & 0.002 & 0.84 \\
\hline$(n=13)$ & Doppler & 100 & 100 & 100 & 100 & 100 & $<0.001$ & 1.000 \\
\hline $13-17$ & Conventional & 100 & 100 & 100 & 100 & 100 & 0.038 & 0.60 \\
\hline$(n=10)$ & Doppler & 100 & 100 & 100 & 100 & 100 & 0.002 & 1.000 \\
\hline
\end{tabular}

PPV: positive predictive value; NPV: negative predictive value

other age-related factors, we analysed the results in 3 separate age groups of 4 to 8 years, 9 to 12 years, and 13 to 17 years. The results indicated that both the colour Doppler and the conventional ultrasound achieved more accurate results at older ages (Table 2).

\section{Discussion}

The findings of this study revealed that conventional ultrasound has a good agreement with the CT scan for the diagnosis of paediatric maxillary sinusitis. If the colour Doppler ultrasound is used, the diagnostic value of this modality will be improved.

Sinusitis is a common paediatric infectious problem. Various diagnostic modalities, such as X-ray, ultrasound, CT scan, and MRI, have been suggested, as the symptoms of sinusitis in children are usually nonspecific $(8,20)$. Few studies have been conducted on the diagnostic value of ultrasounds in identifying sinusitis in children. The present study investigated the diagnostic accuracy of both the conventional ultrasound and colour Doppler ultrasound for the diagnosis of sinusitis in children, compared to the CT scan, which is considered as the gold standard. The findings of this study showed that the conventional ultrasound has a sensitivity of $73.4 \%$, specificity of $100 \%$, positive predictive value of $100 \%$, and negative predictive value of $50.98 \%$ in the diagnosis of sinusitis in children, when compared with CT scan.

When colour Doppler ultrasound was done, it was observed that sensitivity, specificity, and positive and negative predictive values increased, and the values were $89.36 \%, 100 \%, 100 \%$, and $70.58 \%$, respectively. The new points considered in this study were the positive findings of the Doppler ultrasound, which indicated an increase in arterial blood flow velocity, an increase in the diameter of the artery, and a reduced RI in affected sinus, which together were termed as Ghasemi signs.

In a study conducted by Mori et al. on 18 children, ultrasound was compared with CT scan of the sinus for the diagnosis of sinusitis. The sensitivity, specificity, and positive and negative predictive values of ultrasound were $92.6 \%, 100 \%, 100 \%$, and $81.8 \%$, respectively. The authors noted that the diagnostic value of ultrasound for the diagnosis of sinusitis in children is higher than that of the simple X-ray (21).

In 1998, Lichtenstein investigated 100 maxillary sinuses in 50 adult patients in the intensive care unit (ICU); then, he compared the results of the sinus ultrasound (complete, incomplete, and absent sinusogram) with the X-ray and CT scan. He found that the findings of the sinusogram were consistent with those of the CT scan and he presented ultrasound as the first diagnostic tool for sinusitis (22).

Fufezan et al. conducted a study on 67 children to compare the diagnostic values of the X-ray and ultrasound for the diagnosis of maxillary sinusitis. They considered fluid collection and sinus thickening as ultrasound positive findings. Compared with the standard X-ray, ultrasound showed a sensitivity of $94.9 \%$ and specificity of $98.4 \%$. The authors concluded that although ultrasound is not an appropriate technique in diagnosing the thickening of mucosa in paediatric maxillary sinusitis, it is still applicable for confirming fluid collection in the maxillary sinus. The ultrasound findings in this study were compared with X-ray rather than with a CT scan as the gold standard (23).

To the best of our knowledge, to date, no study has been conducted to show the value of the colour Doppler for the diagnosis of paediatric sinusitis. In 2 studies, colour Doppler was found to be valuable in diagnosing and differentiating between purulent maxillary sinusitis and nonpurulent ones. These studies indicated that the presence of dispersed particles due to the maxillary sinus infection can be diagnosed by returning Doppler waves $(15,16)$.

In the current study, RI was considered in paediatric sinusitis for the first time. In a few studies, RI was introduced as an indication of local congestion. $\mathrm{RI}=0.6(0.5-0.7)$ in the test was normal. Dudea et al. demonstrated that this amount is reduced to less than 0.5 in orchitis (17).

In 2 other studies with transcranial Doppler ultrasound, it has been shown that RI is reduced in times of intracranial hyperaemia $(24,25)$. The results of these studies are consistent with those of the present study. In our study, the RI of the affected sinuses was less than 0.5 and was significantly lower than that of the normal sinuses. Given that no studies have been conducted on the diagnostic value of the colour Doppler ultrasound for paediatric sinusitis, it seems that there should be more studies on the merits of this noninvasive procedure.

The sample size was relatively small in this study. We introduced the new sonographic findings as Ghasemi signs for the first time in the diagnosis of paediatric sinusitis. However, each sign must be checked for different age groups and sexes under different test conditions in a larger sample size. Also, we did not have any information about the acute or chronic clinical status of the patients.

On the other hand, we could not design the study to be 
fully blind to the investigators, and this might have been a source of potential bias.

\section{Conclusion}

The findings of this study demonstrated that the conventional ultrasound has a good agreement with CT scan as the gold standard in the diagnosis of paediatric maxillary sinusitis. If colour Doppler is added and the specific findings presented in this study (Ghasemi signs) are considered, the diagnostic value of this modality will be improved.

\section{Conflict of Interests}

The authors declare that they have no competing interests.
21. Mori A, Nakayama T, Tsukidate T, Hirabayashi H, Haruna S. [Comparison of B-mode ultrasonography and computed tomography in the evaluation of maxillary sinusitis in pediatric patients]. Nihon Jibiinkoka Gakkai Kaiho. 2014;117:26-33.

22. Lichtenstein D, Biderman P, Mezière G, Gepner A. The "sinusogram", a real-time ultrasound sign of maxillary sinusitis. Intensive Care Med. 1998;24:1057-61.

23. Fufezan O, Asavoaie C, Cherecheş Panta P, Mihuţ G, Bursaşiu E, Anca I, et al. The role of ultrasonography in the evaluation of maxillary sinusitis in pediatrics. Med Ultrason. 2010;12:4-11

24. Goh D, Minns RA, Pye SD, Steers AJ. Cerebral blood flow velocity changes after ventricular taps and ventriculoperitoneal shunting. Childs Nerv Syst. 1991;7:452-7.

25. Chen KS, Lin KL, Wang HS, Hsia SH, Lin TY, Lin PY. Transcranial Doppler sonography in the early stage of critical enteroviral infection. J Ultrasound Med. 2003;22:1061-6.

\section{References}

1. Bachert C, Hörmann K, Mösges R, Rasp G, Riechelmann H, Müller $\mathrm{R}$, et al. An update on the diagnosis and treatment of sinusitis and nasal polyposis. Allergy. 2003;58:176-91.

2. Bhattacharyya N. Chronic rhinosinusitis: is the nose really involved? Am J Rhinol. 2001;15:169-73.

3. Meltzer EO, Hamilos DL, Hadley JA, Lanza DC, Marple BF, Nicklas RA, et al. Rhinosinusitis: establishing definitions for clinical research and patient care. J Allergy Clin Immunol. 2004;114:155-212.

4. Peters AT, Spector S, Hsu J, Hamilos DL, Baroody FM, Chandra RK, et al. Diagnosis and management of rhinosinusitis: a practice parameter update. Ann Allergy Asthma Immunol. 2014;113:347-85.

5. Fokkens WJ, Lund VJ, Mullol J. European position paper on rhinosinusitis and nasal polyps. Rhinology. 2007;45 suppl 20:1-139.

6. Veskitkul J, Vichyanond P, Pacharn P, Visitsunthorn N, Jirapongsananuruk O. Clinical characteristics of recurrent acute rhinosinusitis in children. Asian Pac J Allergy Immunol. 2015;33:276-80.

7. Ramadan HH. Chronic rhinosinusitis in children. Int J Pediatr/ 2012;2012:573942.

8. Criddle MW, Stinson A, Savliwala M, Coticchia J. Pediatric chronic rhinosinusitis: a retrospective review. Am J Otolaryngol 2008; 29:3728.

9. Lusk R. Pediatric chronic rhinosinusitis. Curr Opin Otolaryngol Head Neck Surg. 2006;14:393-6.

10. Leo G, Triulzi F, Incorvaia C. Sinus imaging for diagnosis of chronic rhinosinusitis in children. Curr Allergy Asthma Rep/ 2012; 12(2):13643

11. Poole MD. A focus on acute sinusitis in adults: changes in disease management. Am J Med. 1999;106:38S-47S.

12. Shahid SK. Rhinosinusitis in children. ISRN Otolaryngol. 2012; 2012:851831.

13. Osguthorpe JD. Adult rhinosinusitis: diagnosis and management. Am Fam Physician. 2001:63:69-76.

14. Puhakka T, Heikkinen T, Mäkelä MJ, Alanen A, Kallio T, Korsoff L, et al. Validity of ultrasonography in diagnosis of acute maxillary sinusitis. Arch Otolaryngol Head Neck Surg. 2000;126:1482-6.

15. Jönsson P, Sahlstrand-Johnson P, Holmer NG, Persson HW, Jannert $M$, Jansson $T$. Feasibility of measuring acoustic streaming for improved diagnosis of rhinosinusitis. Ultrasound Med Bio. 2008;34:228-38.

16. Sahlstrand-Johnson P, Jönsson P, Persson HW, Holmer NG, Jannert $\mathrm{M}$, Jansson T. In vitro studies and safety assessment of Doppler ultrasound as a diagnostic tool in rhinosinusitis. Ultrasound Med Bio. 2010;36:2123-31.

17. Dudea SM, Ciurea A, Chiorean A, Botar-Jid C. Doppler applications in testicular and scrotal disease. Med Ultrason. 2010;12:43-51.

18. Uysal II, Buyukmumcu M, Dogan NU, Seker M, Ziylan T. Clinical significance of maxillary artery and its branches: a cadaver study and review of the literature. Int J Morphol. 2011;29:1274-81.

19. Fleiss JL, Levin B, Paik MC. Statistical methods for rates and proportions. 3. New Jersey: John Wiley \& Sons Inc; 2003.

20. Triulzi F, Zirpoli S. Imaging techniques in the diagnosis and management of rhinosinusitis in children. Pediatr Allergy Immunol. 2007;18 Suppl 18:46-9. 